\title{
MEAN FIELD LIMIT FOR THE ONE DIMENSIONAL VLASOV-POISSON EQUATION.
}

\author{
MAXIME HAURAY
}

\begin{abstract}
We consider systems of $N$ particles in dimension one, driven by pair Coulombian or gravitational interactions. When the number of particles goes to infinity in the so called mean field scaling, we formally expect convergence towards the Vlasov-Poisson equation. Actually a rigorous proof of that convergence was given by Trocheris in Tro86. Here we shall give a simpler proof of this result, and explain why it implies the so-called "Propagation of molecular chaos". More precisely, both results will be a direct consequence of a weak-strong stability result on the one dimensional Vlasov-Poisson equation that is interesting by it own. We also prove the existence of global solutions to the $N$ particles dynamic starting from any initial positions and velocities, and the existence of global solutions to the Vlasov-Poisson equation starting from any measures with bounded first moment in velocity.
\end{abstract}

\section{INTRODUCTION}

1.1. The $N$ particle system. We study the evolution of $N$ particles interacting via Coulombian or gravitational interaction in dimension one. The position and speed of the $i$-th particle will be denoted respectively by $X_{i}^{N}$ and $V_{i}^{N}$, and we will also use the short-cut $Z_{i}^{N}=\left(X_{i}^{N}, V_{i}^{N}\right)$. The large vector containing the positions and speeds of all the particles will be denoted by $\mathcal{Z}^{N}=\left(Z_{i}^{N}\right)_{i \leq N}$.

Our results are valid on the torus and on $\mathbb{R}$, and with (repulsive) Coulombian or (attractive) gravitational interactions. In fact we will neither use the preservation of the energy and the sign of the potential energy, nor the attractive and repulsive character of the force. In that article, we will consider only the case of Coulombian interaction in the periodic domain $\mathbb{T}$, in order to keep the notation as simple as possible. The adaptation to the case of the unbounded domain $\mathbb{R}$ does not raise many difficulties, and it is in some sense simpler, since for instance the interaction potential and force have simpler expression. The adaptation to the gravitational case is also simple.

So in all the sequel, the positions $X_{i}$ belong to $\mathbb{T}$, and the velocities $V_{i}$ to $\mathbb{R}$. On the torus $\mathbb{T}$ that we will identify for simplicity to $\left[-\frac{1}{2}, \frac{1}{2}\right)$, the Coulombian interaction potential $W$ and the associated force $-W^{\prime}$ are given by

$$
W(x):=\frac{x^{2}-|x|}{2}, \quad-W^{\prime}(x):= \begin{cases}-\frac{1}{2}-x & \text { if } x \in\left[-\frac{1}{2}, 0\right), \\ \frac{1}{2}-x & \text { if } x \in\left(0, \frac{1}{2}\right), \\ 0 & \text { if } x=0 .\end{cases}
$$

This particular kernel correspond for instance to the situation where we study electrons in a fixed background of ions. The equality $W^{\prime}(0)=0$ may seem strange since the interaction force is singular at 0 . But, it will be very convenient in the sequel, and will allow for many simplification in the notation. That convention corresponds to the fact that there is no self-interaction. Remark that $W^{\prime}$ may be decomposed in a singular and a non singular part

$$
\text { for }|x| \leq \frac{1}{2}, \quad W^{\prime}(x)=-x+\frac{1}{2} \operatorname{sign}(x), \quad \operatorname{sign}(x):=\frac{x}{|x|} \quad(=0 \text { if } \quad x=0)
$$

2000 Mathematics Subject Classification. 76D05, 65C05.

Key words and phrases. 1D Vlasov-Poisson equation, Propagation of Chaos, Monge-Kantorovich-Wasserstein distance. 
From these formula, we see that the only singularity of the Poisson kernel $W$ is that its second derivative is a Dirac $-\delta_{0}$. But the interaction force $-W^{\prime}$ is globally bounded, so that in this sense it is not so singular. The situation is very different in larger dimensions, where the Coulombian force is always "strongly" singular : $\nabla W \notin L^{d /(d-1)}$ in dimension $d$.

The evolution of the $N$ particles is classically driven by the following system of $N$ second order ODEs

$$
\forall i \leq N, \quad \dot{X}_{i}^{N}=V_{i}^{N}, \quad \dot{V}_{i}^{N}=-\frac{1}{N} \sum_{j \neq i} W^{\prime}\left(X_{i}^{N}-X_{j}^{N}\right) .
$$

Remark that thanks to the assumption that there is no self interaction $\left(W^{\prime}(0)=0\right)$, we may also use a full sum $\sum_{j=1}^{N}$ instead of the more usual $\sum_{j \neq i}$ in the second equation. The $\frac{1}{N}$ factor is not important for a fixed $N$, but it is necessary if we want to obtain the mean field limit when $N$ goes to infinity. It appears when the "physical" system is observed at the appropriate scales of time, position and speed. Here, we will not emphasize more on this point, and we consider uniquely non-dimensional equations in the sequel.

1.2. About the existence of solution to the system of ODEs. As the vector-field that drives this system of ODE is singular (not continous), the existence of solutions to that system is not completely obvious. So to be precise, we shall first define properly the notion of solution that we will use. We only define global (in time) solutions, because we will always deal with such solutions in the sequel.

Definition 1.1. A global solution of the ODE (1.3) with initial condition $\mathcal{Z}^{N}(0)$ is an application $t \mapsto \mathcal{Z}^{N}(t)$ defined on $\mathbb{R}^{+}$such that for all $t \in \mathbb{R}^{+}$, for all $i \leq N$

$$
X_{i}^{N}(t)=X_{i}^{N}(0)+\int_{0}^{t} V_{i}^{N}(s) d s, \quad V_{i}^{N}(t)=V_{i}^{N}(0)-\frac{1}{N} \sum_{j \neq i} \int_{0}^{t} W^{\prime}\left(X_{i}^{N}(s)-X_{j}^{N}(s)\right) d s .
$$

An alternative definition, equivalent to that one, would be to require $\mathcal{Z}^{N}$ to be differentiable almost everywhere in time and (1.3) to hold for all $i \leq N$ and a.e. $t \in \mathbb{R}^{+}$.

In our setting, the now usual theory initiated by DiPerna-Lions [DL89 applies and provides existence and uniqueness of a measure preserving flow. Historically, the first result that can be applied here was given by Bouchut Bou01 : it covers the case of second order ODEs of the previous type with $B V$ force-field. But we may also apply the more general result of Ambrosio Amb04, valid for any ODE with $B V$ vector-field with bounded divergence (in $L^{\infty}$ or even in $L^{1}$ ).

As we shall see later, the uniqueness of the solution to the system of ODEs (1.3) is not important for the mean-field limit, and the existence of solutions will be enough for our work. The existence of a "global" measure preserving flow implies the existence of solutions in the sense of Definition 1.1 for almost all initial conditions. Remark also that the uniqueness of the global measure preserving flow does not implies anything for a particular initial condition. But this is not an issue here since we are not interested by uniqueness.

But here, in our weakly singular setting, global existence of solutions to (1.3) for any initial condition may be obtained thanks to the theory of differential inclusion. Using a result by Filippov [Fil88] we will precisely prove the following Proposition

Proposition 1.2. For any initial configuration $\mathcal{Z}^{N}(0)$, there exists at least one global solution to the system of ODE (1.3) in the sense of Definition 1.1.

The proof of that Proposition is given in Section 2. Thanks to that result, we may now concentrate on the mean-field limit. 
The mean-field limit. In the limit of large number of particles, we assume that the initial distribution of particles converges towards a (maybe smooth) profile $f_{0}$. To measure this convergence, we introduce the empirical measure $\mu_{\mathcal{Z}}^{N}$ defined by

$$
\mu_{\mathcal{Z}}^{N}(t):=\frac{1}{N} \sum_{i=1}^{N} \delta_{Z_{i}^{N}(t)}=\frac{1}{N} \sum_{i=1}^{N} \delta_{\left(X_{i}^{N}(t), V_{i}^{N}(t)\right)} .
$$

We precisely define the convergence towards $f_{0}$ by the convergence in the weak sense of measures of $\mu_{\mathcal{Z}}^{N}$ towards $f_{0}$

$$
\mu_{\mathcal{Z}}^{N}(0) \rightarrow f_{0} \quad * \text {-weakly in } \mathcal{M}(\mathbb{T} \times \mathbb{R}) .
$$

If the distribution of particles also converges at time $t$ towards a distribution profile denoted by $f(t, x, v)$, then we may formally replace in the second equation of (1.3) the discrete sum by an integral of $W^{\prime}$ against the profile $f(t)$ and get a limit ODE, depending of $f$, that drives the limit trajectories of the system

$$
\dot{X}(t)=V(t), \quad \dot{V}(t)=-\int W^{\prime}(X(t)-y) f(t, y, w) d y d w .
$$

From this ODE, using that $f$ must be constant on the trajectories associated to (1.6), we obtain the so-called Vlasov-Poisson equation (that should rather be called "collisionless Boltzmann equation" or "Jeans-Vlasov" equation [Hén82])

$$
\begin{aligned}
& \partial_{t} f+v \partial_{x} f-\partial_{x} \phi(t, x) \partial_{v} f=0, \\
& \phi(t, x)=[W * \rho(t)](x)=\int W(x-y) f(t, y, w) d y d w
\end{aligned}
$$

Here and below we will always refer to $f$ as the distribution of particles (or profile), and to

$$
\rho(t, x):=\int f(t, x, v) d x d v
$$

as the density (in position), always denoted by $\rho$, associated to the full distribution $f$.

The argument above is only a formal justification, but a more rigorous one is given in the following Lemma.

Lemma 1.3. Assume that $\mathcal{Z}^{N}$ is a global solution to the system of ODE (1.3) in the sense of Definition 1.1. Then with the assumption that $W^{\prime}(0)=0$, the associated empirical measure $\mu_{\mathcal{Z}}^{N}$ is a global weak solution to the Vlasov-Poisson equation (1.7).

As usual "weak solution" means here solution in the sense of distribution. We will not write the proof of that property which is classical, at least in the case where $W^{\prime}$ is Lipschitz. We shall just mention that here, we still have a sufficient regularity to perform the requested calculations. In fact, for any smooth $\varphi$, we may use the chain rule to differentiate in time $t \mapsto \varphi\left(t, X_{i}^{N}(t), V_{i}^{N}(t)\right)$ almost everywhere in time. Once the chain rule is applied, we get the result by integration in time and summation on all the particles. Remark that the assumption $W^{\prime}(0)=0$ is crucial here.

That property is interesting because, it helps to rewrite the problem of the mean field limit in a problem of stability of the Vlasov-Poisson equation (1.7). If the sequence of initial empirical measures $\left(\mu_{\mathcal{Z}}^{N}(0)\right)$ converges towards some distribution $f^{0}$, the mean filed limit will holds if :

i) there is a unique solution starting form $f^{0}$ in a suitable class,

ii) that solution is stable (in time) when we approach it by sum of Dirac masses.

In that article, we shall in fact show a "weak-strong" stability principle for the Vlasov-Poisson equation (1.7): if a solution $f$ of the Vlasov equation has a density $\rho$ that remains bounded (uniformly in position) along time, then it is stable in the class of measure solutions with finite first order moment in $v$. Of course, this stability result implies the uniqueness in the class of measures (with finite first moment in $v$ ) of the solution starting from the initial condition $f^{0}$ of $f$. For that reason, that kind of 
result is more commonly called "weak-strong" uniqueness principle. But we choose to emphasize on the stability, that is maybe more important for the mean-field limit.

1.3. Mean-field limit using notation from probability. The system of ODE (1.6) has also an interesting reformulation if we use an idea and a notation borrowed to the theory of probability and more precisely to the theory of continuous stochastic processes. We may say that the $X_{t}=X(t)$ and $V_{t}=V(t)$ appearing in the ODE (1.6) are trajectories of a "non stochastic" process. Even if their is no noise, the probabilistic notation is quite useful for two reasons :

- We can interpret $f^{0}$ as the law of the initial position and speed $\left(X_{0}, V_{0}\right)$,

- the low regularity of the interaction force $W^{\prime}$ implies that the solutions of (1.6) are not unique if $f_{t}$ is not regular enough. This introduces naturally some "randomness", as some choices have to be made for the construction of solutions.

We mention this "probabilistic" point of view was already introduced for (deterministic) ODE by Ambrosio in his study of linear transport and associated ODE with low regularity [Amb04, Section 5] (See also references therein for earlier works with a similar point of view). In fact, we shall use several times a results proved in its well written lecture notes [Amb08]). His ideas were also generalized later by Figalli to the case of SDEs with possibly degenerate noise Fig08.

Here we mostly adopt this point of view in the nonlinear setting, and with a slightly different notation. Precisely, the system (1.6) maybe rewritten as a nonlinear $O D E$. $f_{t}=f(t)$ will be the law at time $t$ of the couple $Z_{t}=\left(X_{t}, V_{t}\right)$ and we use the notation $Z$. to refer to a trajectory, i.e. an element of $C([0,+\infty), \mathbb{T} \times \mathbb{R})$.

Definition 1.4 (Nonlinear ODE). A solution of the non linear ODE below with initial condition $\nu^{0} \in \mathcal{P}(\mathbb{T} \times \mathbb{R})$ is a probability $\mathbb{P}$ on $C([0,+\infty), \mathbb{I} \times \mathbb{R})$ such that $\mathbb{P}\left(Z_{0} \in \cdot\right)=\nu^{0}$ and the following equalities hold for $\mathbb{P} \times \mathbb{P}$-almost every $(Z$., $\bar{Z}$. $)$

$$
\forall t \in[0,+\infty), \quad\left\{\begin{array}{l}
X_{t}=X_{0}+\int_{0}^{t} V_{s} d s, \\
V_{t}=V_{0}-\int_{0}^{t} \mathbb{E}_{\bar{Z} \cdot}\left[W^{\prime}\left(X_{s}-\bar{X}_{s}\right)\right] d s .
\end{array}\right.
$$

If as usual, we prefer to speak with random variables than with laws, we must just say that $Z$. is a random variable, that the law of $Z_{0}$ is $\nu_{0}$, and that the previous equation are satisfied with $\bar{Z} .=(\bar{X} . \bar{V}$. $)$ an independent copy of $Z$., i.e. a independent process with same law than $Z$. . Remark that as in the previous system of ODEs, the requirement of equation (1.8) is equivalent to ask that the associated equality on $\dot{X}_{t}$ and $\dot{V}_{t}$ are true almost surely and for almost all time $t \in[0,+\infty)$.

In fact this definition is just a reformulation of the system (1.6), with expectation instead of integral, and with the vocabulary of stochastic processes. A process $Z$. solution to (1.8) is concentrated on deterministic solutions to (1.6). But it has some interest when we will use optimal transport and coupling techniques. The probabilistic notation in which the effective probability spaces and optimal mappings are never explicitly written is sometimes more simple to handle. It also allows to work directly on trajectories with a simple notation.

We refer to (1.8) as a nonlinear ODE, in reference to the nonlinear SDEs introduced by McKean in McK66, also known as McKean-Vlasov processes. These nonlinear SDEs correspond to add a brownian drift (or more generally a random force field) in the second equation of (1.8), and possibly the action of an exterior potential. As in our deterministic case, these nonlinear SDEs are natural limits of N particle systems with noise McK67, Mél96.

In the case of smooth interactions, the so-called methods of characteristics implies that the VlasovPoisson equation (1.7) and associated the nonlinear ODE (1.4) give exactly the same solutions. In fact, when the force field is sufficiently regular for uniqueness of the trajectories to hold, the two problems are equivalent. With non smooth interactions, the uniqueness of trajectories is lost and we are only able to state the following lemma. 
Lemma 1.5. Assume that $Z$. is a solution of the nonlinear ODE (1.8). Then, the law $\nu_{t}$ of its time marginals $Z_{t}$ is a weak solution of the Vlasov-Poisson equation (1.7).

Reciprocally, if $\nu_{t}$ is a bounded measure solution to the Vlasov-Poisson equation (1.7), there exists a process $Z$. solution to (1.8) whose time marginals are exactly the $\nu_{t}$.

Without noise, the first part of this lemma is just an application of the chain rule. The second part of the lemma is more delicate. It is a consequence of a theorem of Ambrosio, for linear transport equation. In fact $\nu_{t}$ is a solution of the linear transport equation

$$
\partial_{t} \mu+v \partial_{x} \mu+E\left[\nu_{t}\right] \partial_{v} \mu=0
$$

where the fixed force field is given by $E\left[\nu_{t}\right]:=-\int W^{\prime}(x-y) \nu_{t}(d y, d w)$. In that setting, we can apply Amb08, Theorem 3.2] to obtain a process $Z$. solution to (1.8) with time marginals $\nu_{t}$.

Another interesting property associated to these nonlinear ODE is the following

Lemma 1.6. If $\mathcal{Z}^{N}$ is a solution to the $N$ particles system (1.3), then the empirical measure on the trajectories

$$
\mathbb{P}_{\mathcal{Z} .}^{N}:=\frac{1}{N} \sum_{i=1}^{N} \delta_{Z_{i,}^{N}}
$$

is a solution of the nonlinear $O D E$ (1.8) with initial condition $\mu_{\mathcal{Z}}^{N}(0)$.

As for Lemma 1.3, this is more a way of rewriting the equations (1.3), and we will not be more precise. Note that there is no reciprocal statement : along solutions of the nonlinear ODE (1.8) starting from sum of Dirac masses, the singularity of the Force allows to split some Dirac masses in smaller ones and also in less singular measures supported on a line MMZ94a, MMZ94b, DFVar.

Using approximation by sum of Dirac masses (a strategy already used in aggregation equation, see $\mathrm{CDF}^{+} 11$ and references therein), we will be able to give another proof of a general existence theorem for the one-dimensional VP equation obtained by Zheng and Majda [ZM94

Theorem 1.7. Assume that $\nu^{0}$ is a probability measure with finite first moment in velocity : $\int|v| \nu^{0}(d x, d v)<$ $\infty$, and recall that we use the convention $W^{\prime}(0)=0$. Then there exists (at least one) global solution to the Vlasov equation (1.7), with initial condition $\nu^{0}$, and also one process solution of the nonlinear $O D E$ (1.8) with the same initial condition.

The proof of Theorem 1.7 is done in Section 2 Here we will only make some comments :

- In order to define properly the interaction force (which has to be defined everywhere if we want to define measure solutions) we have introduced the convention $W^{\prime}(0)=0$. Madja and Zheng [ZM94] use a strategy that may seem different, but both are in fact equivalent.

- Our result is more general that the one of Zheng and Majda, which requires a exponential moment (in $v$ ) on the initial measure $\nu^{0}$.

- Our proof is also in some sense more simple. It relies on the approximation of the initial data by sum of Dirac masses, for which we known by Proposition 1.2 that there exists global solutions to (1.3) and thus (1.8). Then, the tightness of the law of these processes is obtained rather easily, but more work is required to characterize the limit. For the last point, we first proof that the time marginals associated to any limit process are solution of the VP equation (1.7). Once the time marginals, and therefore the force field are known, we can apply the second point of Lemma 1.5 to get the existence of a process solution to (1.8) with the requested time marginals. But we were not able to prove that the limit process is a solution to the nonlinear ODE (1.8). Of course, the use of the nonlinear ODE (1.8) is not mandatory if we are interest only by the result on the Vlasov-Poisson equation, but it will nevertheless be convenient, since we will need to perform some estimates on the trajectories. 
1.4. A weak-strong stability estimate around solutions to VP equation with bounded density. Here we will state the main result of our article : a weak-strong stability estimate around solutions of the Vlasov-Poisson equation (1.7) with bounded density. Recall that in this article we use the word "density" only to refer to the density in physical space $\rho(t, x)=\int f(t, x, v) d v$.

We introduce the Monge-Kantorovitch-Wasserstein distance of order one, denoted by $W_{1}$ constructed with the Euclidian distance on $\mathbb{T} \times \mathbb{R}$. We refer to the clear book of Villani [Vil03] for an introduction to this object. We will use it both with random variables or their law (which are probabilities) in the argument, depending of the situation.

Theorem 1.8. Assume that $Z^{1}$ is a solution of the nonlinear ODE (1.8), with time marginals $f_{t}$ that have bounded density $\rho_{t}$ for any time $t \geq 0$. Then for any global solution $Z^{2}$ of the nonlinear $O D E$ (1.8) with finite first order moment in $v$, we have the following stability estimate

$$
\forall t \in \mathbb{R}^{+}, \quad W_{1}\left(Z_{t}^{1}, Z_{t}^{2}\right) \leq e^{a(t)} W_{1}\left(Z_{0}^{1}, Z_{0}^{2}\right), \quad \text { with } \quad a(t):=\sqrt{2} t+8 \int_{0}^{t}\left\|\rho_{s}\right\|_{\infty} d s .
$$

Theorem 1.9. Assume that $f_{t}$ is a solution of the Vlasov Poisson equation (1.7) with bounded density $\rho_{t}$ for any time $t \geq 0$. Then for any global measure solution $\nu$ to the same equation with finite first order moment in $v$, we have the following stability estimate

$$
\forall t \in \mathbb{R}^{+}, \quad W_{1}\left(f_{t}, \nu_{t}\right) \leq e^{a(t)} W_{1}\left(f^{0}, \nu_{0}\right), \quad \text { with } \quad a(t):=\sqrt{2} t+8 \int_{0}^{t}\left\|\rho_{s}\right\|_{\infty} d s .
$$

Theorem 1.9 implies Theorem [1.8, since time marginals of solutions of the nonlinear ODE (1.8) are solutions of the Vlasov-Poisson equation (1.7). But, the converse is also true, since thanks to the second part of Lemma 1.5, any solution of the VP equation may be represented as time marginals of a solution of the nonlinear ODE. In view of that, we will only proof Theorem 1.8 in section 2 But remark also that this proof written with the formalism of processes maybe adapted to the formalism of solution of Vlasov -Poisson equation (1.7), with less simple notation (in our opinion). So, again, the choice of working with VP equation of the nonlinear ODE is rather a matter of convenience.

1.5. Existence of strong solutions. The later result is interesting only if solutions with bounded density do exist. But, this is a well-known fact [CK80, Bos05, Guo95, LMR10. Here we will restate a Proposition about the existence of such solutions that relies precisely on Theorem 1.8 or 1.9. Remark that theorem 1.9 implies that such solutions are automatically unique.

Proposition 1.10. Assume that $f_{0}$ satisfies

$$
\int_{<} 0^{+\infty} g_{0}(v) d v<+\infty, \quad\left\|f_{0}\right\|_{\infty}=g(0)<+\infty, \quad \text { where } \quad g_{0}(v):=\sup _{y \in \mathbb{T},|w| \geq v}\left|f_{0}(x, w)\right| .
$$

Then there exists one unique solution $Z$. to the nonlinear ODE (1.8), with initial condition $f^{0}$. There also exists a unique solution to the Vlasov-Poisson equation (1.7) with initial condition $f^{0}$, given by the time marginals $f_{t}$ of $Z$.

The density (in position) $\rho_{t}$ associated to $f_{t}$ satisfies in particular the bound

$$
\left\|\rho_{t}\right\|_{\infty} \leq 2 \int_{0}^{+\infty} g_{0}(v) d v+\left\|f_{0}\right\|_{\infty} t
$$

1.6. Mean-field limit. If the initial condition $f_{0}$ satisfies the condition (1.9), the mean-field limit around the unique solution $f$ starting from $f_{0}$ is a direct consequence of Theorem [1.9. In fact, it suffice to apply this theorem to the particular case of initial data $\nu_{0}$ given by sum of $N$ Dirac masses. This is precisely stated in the following corollary, for which we will not give a specific proof.

Corollary 1.11 (of Theorem 1.9). Assume that $f_{0}$ satisfies the condition (1.9) and denote by $f_{t}$ the unique solution of the VP equation (1.7) with initial condition $f_{0}$. Proposition 1.10 ensures that its 
density $\rho_{t}$ is bounded. For any $\mathcal{Z}^{N}$ solution of the $N$ particles system (1.3) with initial empirical measure $\mu_{\mathcal{Z}}^{N}(0)$, we have the following stability estimate

$$
W_{1}\left(\mu_{\mathcal{Z}}^{N}(t), f_{t}\right) \leq e^{a(t)} W_{1}\left(\mu_{\mathcal{Z}}^{N}(0), f_{0}\right) \quad \text { with } \quad a(t):=\sqrt{2} t+8 \int_{0}^{t}\left\|\rho_{s}\right\|_{\infty} d s .
$$

This implies the mean-field limit in large number of particles since if the positions and velocities of the particles are chosen so that

$$
\mu_{\mathcal{Z}}^{N}(0) \underset{N \rightarrow+\infty}{\longrightarrow} f^{0} \text { weakly, } \quad \sup _{n \in \mathbb{N}} \int|v| \mu_{\mathcal{Z}}^{N}(0, d x d v) \rightarrow \int|v| f_{0}(d x, d v),
$$

then $W_{1}\left(\mu_{\mathcal{Z}}^{N}(0), f_{0}\right) \rightarrow 0$ (See Vil03]). The above corollary implies that the weak convergence holds at any time. Remark that we may also obtain a similar result on the trajectories, i.e. on the solutions of the nonlinear ODE (1.8).

1.7. Propagation of molecular chaos. Around profiles with bounded density, the propagation of chaos is also a consequence of the "weak-strong" stability theorem.

Corollary 1.12 (of Theorem [1.9). Assume that $f^{0}$ satisfies the condition (1.9). Then with the same notation than in Corollary 1.11, we have

$$
\mathbb{E}\left[W_{1}\left(\mu_{\mathcal{Z}}^{N}(t), f_{t}\right)\right] \leq e^{a(t)} \mathbb{E}\left[W_{1}\left(\mu_{\mathcal{Z}}^{N}(0), f_{0}\right)\right]
$$

We shall not give the proof of that results, which is obtain by taking the expectation in (1.10). We just make some comments :

- it is also possible to obtain other related results, for instance bounds on the exponential moments of $W_{1}\left(\mu_{\mathcal{Z}}^{N}(t), f_{t}\right)$ can be obtain in terms of exponential moments of $W_{1}\left(\mu_{\mathcal{Z}}^{N}(0), f_{0}\right)$, which are known to exists, see for instance Boi11,

- the result may be adapted to the "language" of nonlinear ODE,

- our results are stated in terms of convergence in law of the empirical measure towards $f_{t}$. For the relations to the more usual convergence of $k$-particles marginals $(k \geq 2)$, we refer for instance to the famous lecture notes by Sznitman Szn91. We also mentioned that quantitative equivalence estimates were also recently obtained in [MM11] and HM12.

\section{Proofs of the Main Results}

2.1. Proof of Proposition 1.2. The existence will be proved with the help of the theory of differential inclusion Fil88. First we will construct an associated differential inclusion, to which solutions do exist, and then prove that these solutions are indeed solutions to the original problem.

Step 1. The construction of an adapted differential inclusion.

We will replace the system of equation (1.3) by a differential inclusion

$$
\dot{\mathcal{Z}}^{N}(t) \in \mathcal{B}^{N}\left(\mathcal{Z}^{N}(t)\right)
$$

where $\mathcal{B}^{N}$ is set-valued, i.e. is an application from $\mathbb{R}^{2 N}$ into $\mathcal{P}\left(\mathbb{R}^{2 N}\right)$. A Theorem by Filippov [Fil88, Chapter 2, Theorem 1] ensures the existence of global solutions to the differential inclusion (2.11) for any initial condition if :

- for each $\mathcal{Z}^{N} \in \mathbb{R}^{2 N}$, the set $\mathcal{B}^{N}\left(\mathcal{Z}^{N}\right)$ is bounded, and closed, and convex,

- $\mathcal{B}^{N}$ is locally bounded, i.e. for any compact set $K \in \mathbb{R}^{2 N}$ there exists a compact set $K^{\prime} \in \mathbb{R}^{2 N}$ such that $\mathcal{B}^{N}\left(\mathcal{Z}^{N}\right) \subset K^{\prime}$ for all $\mathcal{Z}^{N} \in K$,

- $\mathcal{B}^{N}$ is upper continuous with respect to the inclusion.

A natural $\mathcal{B}^{N}$ may be constructed as follow. We will not write the full application $\mathcal{B}^{N}$, but assume that it has the form

$$
\dot{\mathcal{Z}}^{N}(t) \in \mathcal{B}^{N}\left(\mathcal{Z}^{N}(t)\right) \Leftrightarrow \dot{X}_{i}^{N}=V_{i}, \quad \dot{V}_{i}=\frac{1}{N} \sum_{i} F_{i j}^{N}
$$


where the forces $F_{i j}^{N}$ satisfy some conditions to be specified.

A first choice for the $F_{i j}^{N}$ is to ask that for all $i, j \leq N$ :

- $F_{i j}^{N}=-W^{\prime}\left(X_{i}^{N}-X_{j}^{N}\right)$ when $X_{i}^{N} \neq X_{j}^{N}$,

- $F_{i j}^{N} \in\left[-\frac{1}{2}, \frac{1}{2}\right]$ when $X_{i}^{N}=X_{j}^{N}$.

The associated $\mathcal{B}^{N}$ satisfies all the required properties, but unfortunately, the associated solutions will not be solution of the original ODE system in general. For instance if you take two particles, both originally at position 0 , with velocity 0 , then according to (1.3) and the fact that $W^{\prime}(0)=0$, they should never move. But with our construction of $\mathcal{B}^{N}$, the solution where the two particles remain stuck together and accelerate uniformly with acceleration $\frac{1}{2}$ is admissible.

To overcome this problem, we should define $\mathcal{B}^{N}$ almost as before, but with the additional conditions that we want the action-reaction principle to be valid in any case. The precise conditions are that for all $i<j \leq N$ :

- $F_{i j}^{N}=-F_{j i}^{N}=-W^{\prime}\left(X_{i}^{N}-X_{j}^{N}\right)$ when $X_{i}^{N} \neq X_{j}^{N}$, - $F_{i j}^{N}=-F_{j i}^{N} \in\left[-\frac{1}{2}, \frac{1}{2}\right]$ when $X_{i}^{N}=X_{j}^{N}$.

It is not difficult that to see that this $\mathcal{B}^{N}$ still fulfils the requested properties, and [Fil88, Chapter 2, Theorem 1] ensures the existence of a solution also denoted $\mathcal{Z}^{N}$ to the associated differential inclusion.

Step 2. Solutions of the differential inclusion are solutions to the $N$ particles problem.

According to the final definition of $\mathcal{B}^{N}$, we see that problems may occur only when two particles are at the same position. Here, we will only deal with particles 1 and 2 , the others case being similar. But since for any $t \in \mathbb{R}^{+}$and any $\lambda>0$, the set $\left\{s \in[0, t]\right.$ s.t. $X_{1}^{N}(s)=X_{2}^{N}(s)$ and $\left.\left|V_{1}^{N}(s)-V_{1}^{N}(s)\right| \geq \lambda\right\}$, is made of isolated points, we have also that the set

$$
A_{t}:=\left\{s \in[0, t] \text { s.t. } X_{1}^{N}(s)=X_{2}^{N}(s) \text { and } V_{1}^{N}(s) \neq V_{2}^{N}(s)\right\}
$$

is negligible with respect to the Lebesgue measure. Since the exact value of the force used on negligible set has no influence on the solution, it remains only to understand what happens on the set

$$
B_{t}:=\left\{s \in[0, t] \text { s.t. } X_{1}^{N}(s)=X_{2}^{N}(s) \text { and } V_{1}^{N}(s)=V_{1}^{N}(s)\right\}
$$

For this set, we will use the following lemma, that we shall prove later.

Lemma 2.1. Assume that $g:[0, t] \rightarrow \mathbb{R}$ is a Lipschitz function (differentiable almost everywhere by the Rademacher theorem). Then the set

$$
\left\{s \in[0, t] \text { s.t. } g(0)=0 \text { and } g^{\prime}(0) \neq 0\right\} \quad \text { is negligible. }
$$

Applying this Lemma with the function $g(s)=V_{1}^{N}(s)-V_{2}^{N}(s)$ we get that the set

$$
\left\{s \in[0, t] \text { s.t. } X_{1}^{N}(s)=X_{2}^{N}(s) \text { and } V_{1}^{N}(s)=V_{1}^{N}(s) \text { and }\left(V_{1}^{N}\right)^{\prime}(s) \neq\left(V_{1}^{N}\right)^{\prime}(s)\right\}
$$

is negligible. Then the only part we have really to deal with is

$$
C_{t}:=\left\{s \in[0, t] \text { s.t. } X_{1}^{N}(s)=X_{2}^{N}(s) \text { and } V_{1}^{N}(s)=V_{1}^{N}(s) \text { and }\left(V_{1}^{N}\right)^{\prime}(s)=\left(V_{1}^{N}\right)^{\prime}(s)\right\} \text {. }
$$

But remark that for $s \in C_{t}$ the particles are at the same position and are submitted to the same global force. If no other particle share their common position at this time, it follows from the (final) definition of $\mathcal{B}^{N}$ that $F_{12}^{N}=-F 21^{N}=0$. If there is another particle that share their common position, the situation is a little bit different. Say for instance that the third particles is also at the same position. Then, the configuration where $F_{i, i+1}=-F_{i+1, i}=\frac{1}{2}$ (whit the convention $3+1=1$ ) is admissible. In that case, $F_{12} \neq 0$, but it is also clear that we can set all the forces $F_{i, i+1}$ to zero without changing anything to all the global forces seen by the three particles.

In general, since the particle 1 and 2 are submitted to the same force at time $s$ (remember that $s \in C_{t}$ ), and because the action-reaction principle is preserved by the differential inclusion, this common force is also the one that applies to their center of mass. And in the case where $F_{12}(s) \neq 0$, 
we will not change the solution if we replace the $F_{i j}^{N}$ by the force $\tilde{F}_{i j}^{N}$ acting on their centrer of mass, defined by

$$
\tilde{F}_{12}=0, \quad \tilde{F}_{1 i}=\tilde{F}_{2 i}=-\tilde{F}_{i 1}=-\tilde{F}_{i 2}=\frac{1}{2}\left(F_{1 i}+F_{2 i}\right) \text { for } i \geq 3 .
$$

In fact that replacement is acceptable by the convexity of $\mathcal{B}^{N}$, and this modification does not affect the global force seen by each particle. It means that the original equation(1.3) is already fulfilled at this time $s$.

Finally, we have proved that the system of equations (1.3) is satisfied for almost all time and for all couple $i \neq j$. As already said, this is equivalent to the definition 1.1

Proof of Lemma 2.1. For simplicity we will assume that $g$ has a Lipschitz constant equals to one. If for all $\lambda>0$, the set $A_{\lambda}:=\left\{s \in[0, t]\right.$ s.t. $g(0)=0$ and $\left.g^{\prime}(0) \geq \lambda\right\}$ is negligible, and if also a similar statement is true for the negative value of $g^{\prime}$, then it is not difficult to conclude.

So we will prove by contradiction that $A_{\lambda}$ is of zero measure for any $\lambda>0$. If not, then we may choose a $\lambda>0$ such that $A_{\lambda}$ has strictly positive measure. It is then classical that $A_{\lambda}$ possesses an accumulation point $s$ (See for instance [Ste70, in particular for the argument with non centered interval), for which

$$
\lim _{\varepsilon, \varepsilon^{\prime} \rightarrow 0} \alpha\left(\varepsilon, \varepsilon^{\prime}\right)=1, \quad \text { where } \alpha\left(\varepsilon, \varepsilon^{\prime}\right):=\frac{\mid\left\{u \in\left[s-\varepsilon, s+\varepsilon^{\prime}\right] \text { s.t. } g(0)=0 \text { and } g^{\prime}(0) \geq \lambda\right\} \mid}{\varepsilon+\varepsilon^{\prime}}
$$

But we also have

$$
\begin{aligned}
g\left(s+\varepsilon^{\prime}\right)-g(s-\varepsilon) & =\int_{s-\varepsilon}^{s+\varepsilon^{\prime}} g^{\prime}(r) d r \geq\left(\varepsilon+\varepsilon^{\prime}\right)\left[\lambda \alpha\left(\varepsilon, \varepsilon^{\prime}\right)-\left(1-\alpha\left(\varepsilon, \varepsilon^{\prime}\right)\right)\right] \\
& \geq\left(\varepsilon+\varepsilon^{\prime}\right)\left[(1+\lambda) \alpha\left(\varepsilon, \varepsilon^{\prime}\right)-1\right], \\
& \geq \frac{\lambda}{2}\left(\varepsilon+\varepsilon^{\prime}\right) \quad \text { for all } \varepsilon, \varepsilon^{\prime} \text { small enough. }
\end{aligned}
$$

But this contradicts the fact that $s$ is an accumulation point for $A_{\lambda}$.

2.2. Proof of Theorem 1.7. The proof is separated in five steps. In the first one, we construct an approximating sequence made of solutions to the $\mathrm{N}$ particles systems. In the second, we show the tightness of that sequence, and in the last three ones, we show that the time marginals of the limit process are solution of the Vlasov-Poisson equation (1.7). Afterthat, the existence of a process solution to the nonlinear ODE (1.8) can be obtained by an application of the second point of Lemma 1.5 .

Step 1. Construction of the approximating sequence.

This is quite simple. For each $\mathrm{N}$ choose a sequence $\mathcal{Z}_{0}^{N}$ of initial positions and velocities, such that the associated empirical measures $\mu_{0}^{N}=\mu_{\mathcal{Z}}^{N}(0)$ converges weakly towards the measure $\nu_{0}$. Since $\int|v| \nu_{0}(d x, d v)<+\infty$, we may also assume that

$$
\sup _{N \in \mathbb{N}} \int|v| \mu_{\mathcal{Z}}^{N}(0, d x, d v)<+\infty
$$

Applying Proposition 1.2, we obtain a global solution $\mathcal{Z}^{N}$ to the system of ODE (1.3). Then Lemma 1.6 ensure that it also define a process denoted by $Z_{.}^{N}$, solution to the nonlinear ODE (1.8) with initial condition $\mu_{\mathcal{Z}}^{N}(0)$.

Step 2. Tightness of the approximating sequence. Using the equation (1.4), we get that for almost all $(s, u)$ satifying $0<s<u<t$

$$
\left|V_{u}^{N}-V_{s}^{N}\right| \leq \int_{s}^{u} \mathbb{E}_{\bar{Z} \cdot}\left[\left|W^{\prime}\left(X_{r}^{N}-\bar{X}_{r}^{N} \mid\right] d r \leq \frac{1}{2}\right| u-s \mid .\right.
$$


And with the particular choice $s=0$, we obtain the bound $\left|V_{u}^{N}\right| \leq\left|V_{0}^{N}\right|+\frac{u}{2}$. That bound allows to obtain a similar estimate for the position

$$
\left|X_{u}^{N}-X_{s}^{N}\right| \leq \int_{s}^{u}\left|V_{r}^{N}\right| d r \leq\left(\left|V_{0}^{N}\right|+\frac{t}{2}\right)|s-u| .
$$

Merging estimates (2.13) and (2.14) and taking the supremum on all couple $(u, s)$ we get

$$
\sup _{s, u \in[0, t]} \frac{\left|Z_{u}^{N}-Z_{s}^{N}\right|}{|u-s|} \leq\left|V_{0}^{N}\right|+\frac{t+1}{2} .
$$

Taking the expectation and using (2.12), we get that

$$
\sup _{N \in \mathbb{N}} \mathbb{E}\left[\sup _{s, u \in[0, t]} \frac{\left|Z_{u}^{N}-Z_{s}^{N}\right|}{|u-s|}\right]<+\infty
$$

This implies the tightness. In fact, by the Arzelà-Ascoli theorem, the subsets $\mathcal{B}_{R}^{\lambda}(t)$ of $C([0, t], \mathbb{T} \times \mathbb{R})$ defined by

$$
\mathcal{B}_{R}^{\lambda}(t):=\left\{Z \text {. s.t. }\left|X_{0}\right| \leq R \text { and }\left\|Z^{\prime}\right\|_{\infty} \leq \lambda\right\}
$$

are compact for all $R, \lambda, t \in \mathbb{R}^{+}$. But the fact that $\mathbb{P}\left(Z_{0}^{N} \in \cdot\right)=\mu_{0}^{N}$, together with estimates (2.12) and (2.15) imply that the compact sets $\mathcal{B}_{R}^{\lambda}(t)$ are almost of full measure when $R$ and $\lambda$ are large.

Therefore, up to the extraction of a subsequence, we may assume that the sequence of processes $\left(Z^{N}\right)$ converges weakly towards a process $Z$. . To understand this properly, we must say that $C([0,+\infty), \mathbb{T} \times \mathbb{R})$ is endowed with the topology of uniform convergence on every bounded time interval.

Remark also that the processes $Z_{.}^{N}$ are concentrated on the set of trajectories such that $t \mapsto V_{t}^{N}$ are uniformly Lipschitz with constant $\frac{1}{2}$, a set that is closed under the topology of uniform convergence on any bounded time interval. So this is also true for the process $Z$. : almost surely, the velocity component of the limit process $Z$. has Lipschitz trajectories, with constant $\frac{1}{2}$.

Step 3. Characterization of the limit process $Z$..

First by construction it is clear that the limit process $Z$. satisfies the required initial condition. Next we will show that its time marginals satisfy the Vlasov-Poisson equation (1.7). For this choose two times $s<t$ and a smooth function $\varphi$. We need to show that

$$
\begin{array}{r}
\mathbb{E}_{(Z ., \bar{Z} .)}\left[\left(\varphi\left(t, Z_{t}\right)-\varphi\left(s, Z_{s}\right)-\int_{s}\left[\partial_{t} \varphi\left(u, Z_{u}\right)+V_{s} \partial_{x} \varphi\left(u, Z_{u}\right)\right.\right.\right. \\
\left.\left.\left.-W^{\prime}\left(X_{u}-\bar{X}_{u}\right) \partial_{v} \varphi\left(u, Z_{u}\right)\right] d u\right)\right]=0 .
\end{array}
$$

since $Z^{N}$ are solution of the nonlinear ODE (1.8), it is clear that this is satisfied if we replace $Z$. by $Z^{N}$. Because of the continuity of $\varphi$, we can use classical results, see Billingsley [Bil99, p. 30], and pass to the limit in

$$
\mathbb{E}_{\left(Z^{N}, \bar{Z}^{N}\right)}\left[\varphi\left(t, Z_{t}^{N}\right)-\varphi\left(s, Z_{s}^{N}\right)\right] \underset{N \rightarrow+\infty}{\longrightarrow} \mathbb{E}_{(Z ., \bar{Z} .)}\left[\varphi\left(t, Z_{t}\right)-\varphi\left(s, Z_{s}\right)\right] .
$$

In fact, only the term containing $W^{\prime}$ is more involved, since the interaction force is discontinuous at the origin. To control it, we need to separate the distant and close interactions. For this, we choose an $\varepsilon>0$ and introduce a smooth cut-off function $\chi_{\varepsilon}$ defined on $\mathbb{T}$ and satisfying (we use again the identification $\mathbb{T}=\left[-\frac{1}{2}, \frac{1}{2}\right)$ )

$$
\chi_{\varepsilon}(x)=1 \text { if }|x| \leq \varepsilon, \quad \chi_{\varepsilon}(x)=0 \text { if }|x| \geq 2 \varepsilon .
$$


Then, we can decompose

$$
\begin{aligned}
\left.\mathbb{E}_{\left(Z^{N}, \bar{Z}^{N}\right)}\left[\int_{s}^{t} W^{\prime}\left(X_{u}^{N}-\bar{X}_{u}^{N}\right) \partial_{v} \varphi\left(u, Z_{u}^{N}\right)\right] d u\right] \\
\left.=\mathbb{E}_{\left(Z^{N}, \bar{Z}^{N}\right)}\left[\int_{s}^{t} W^{\prime}\left(X_{u}^{N}-\bar{X}_{u}^{N}\right)\left(1-\chi_{\varepsilon}\left(X_{u}^{N}-\bar{X}_{u}^{N}\right)\right) \partial_{v} \varphi\left(u, Z_{u}^{N}\right)\right] d u\right] \\
\left.\quad+\mathbb{E}_{\left(Z^{N}, \bar{Z}^{N}\right)}\left[\int_{s}^{t} W^{\prime}\left(X_{u}^{N}-\bar{X}_{u}^{N}\right) \chi_{\varepsilon}\left(X_{u}^{N}-\bar{X}_{u}^{N}\right) \partial_{v} \varphi\left(u, Z_{u}^{N}\right)\right] d u\right] .
\end{aligned}
$$

In the first term of the r.h.s that take into account the interactions between distant particles, everything is now continuous. So, as before, it is not difficult to pass in the limit in that term. It converges towards

$$
\left.\mathbb{E}_{(Z, \bar{Z} .)}\left[\int_{s}^{t} W^{\prime}\left(X_{u}-\bar{X}_{u}\right)\left(1-\chi_{\varepsilon}\left(X_{u}-\bar{X}_{u}\right)\right) \partial_{v} \varphi\left(u, Z_{u}\right)\right] d u\right]
$$

It remains to control the second term. We will show that it goes to zero as $\varepsilon$ goes to zero, uniformly in $N$, and that this convergence also holds for the limit process $Z$. . This will conclude the proof.

For this we choose a $\beta<\frac{1}{2}$ and introduce a second smooth cut-off function defined on $\mathbb{R}$, such that

$$
\xi_{\varepsilon}(v)=1 \text { if }|v| \leq \varepsilon^{\beta}, \quad \xi_{\varepsilon}(v)=0 \text { if }|v| \geq 2 \varepsilon^{\beta} .
$$

We use it to separate the remaining term in two : one term taking into account the close interactions with large relative speed, and the second one taking into account the close interactions with small relative speed

$$
\begin{aligned}
\left.\mathbb{E}_{\left(Z^{N}, \bar{Z}^{N}\right)}\left[\int_{s}^{t} W^{\prime}\left(X_{u}^{N}-\bar{X}_{u}^{N}\right) \chi_{\varepsilon}\left(X_{u}^{N}-\bar{X}_{u}^{N}\right) \partial_{v} \varphi\left(u, Z_{u}^{N}\right)\right] d u\right] \\
\left.=\mathbb{E}_{\left(Z^{N}, \bar{Z}^{N}\right)}\left[\int_{s}^{t} W^{\prime}\left(X_{u}^{N}-\bar{X}_{u}^{N}\right) \chi_{\varepsilon}\left(X_{u}^{N}-\bar{X}_{u}^{N}\right)\left(1-\xi_{\varepsilon}\left(V_{u}^{N}-\bar{V}_{u}^{N}\right)\right) \partial_{v} \varphi\left(u, Z_{u}^{N}\right)\right] d u\right] \\
\left.\quad+\mathbb{E}_{\left(Z^{N}, \bar{Z}^{N}\right)}\left[\int_{s}^{t} W^{\prime}\left(X_{u}^{N}-\bar{X}_{u}^{N}\right) \chi_{\varepsilon}\left(X_{u}^{N}-\bar{X}_{u}^{N}\right) \xi_{\varepsilon}\left(V_{u}^{N}-\bar{V}_{u}^{N}\right) \partial_{v} \varphi\left(u, Z_{u}^{N}\right)\right] d u\right] .
\end{aligned}
$$

Step 4. The effects of close interaction with large relative speed. To understand the first term in the r.h.s. of the above decomposition, we choose a particular couple of trajectories, denoted by $\left(Z^{N}, \bar{Z}^{N}{ }^{N}\right.$ ) (the notation is the same than for processes, which are probabilities on trajectories, but we will always explicitly mention which case we are referring to). Since $\partial_{v} \varphi$ and $W^{\prime}$ are bounded, we may write

$$
\begin{gathered}
\left.\mid \int_{s}^{t} W^{\prime}\left(X_{u}^{N}-\bar{X}_{u}^{N}\right) \chi_{\varepsilon}\left(X_{u}^{N}-\bar{X}_{u}^{N}\right)\left(1-\xi_{\varepsilon}\left(V_{u}^{N}-\bar{V}_{u}^{N}\right)\right) \partial_{v} \varphi\left(u, Z_{u}^{N}\right)\right] d u|\leq C| A\left(Z^{N}, \bar{Z}^{N}\right) \mid \\
\text { where } A\left(Z^{N}, \bar{Z}^{N}\right):=\left\{u \in[s, t] \text { s.t. }\left|X_{u}^{N}-\bar{X}_{u}^{N}\right|<2 \varepsilon \text { and }\left|V_{u}^{N}-\bar{V}_{u}^{N}\right|>\varepsilon^{\beta}\right\} .
\end{gathered}
$$

We used the notation $|A|$ to denotes the Lebesgue measure of any measurable subset $A$ of $\mathbb{R}$. Now, we pick up a time $r \in A\left(Z^{N}, \bar{Z}^{N}\right)$. Since $V^{N}$ and $\bar{V}^{N}$ are almost surely Lipschitz with constant $\frac{1}{2}$, we may write that

$$
\forall u \in I_{r}^{N}:=\left[r, r+\frac{\left|V_{r}^{N}-\bar{V}_{r}^{N}\right|}{2}\right], \quad\left|V_{u}^{N}-\bar{V}_{u}^{N}\right| \in\left[\frac{\left|V_{r}^{N}-\bar{V}_{r}^{N}\right|}{2}, \frac{3\left|V_{r}^{N}-\bar{V}_{r}^{N}\right|}{2}\right],
$$

and in particular, the sign of the relative speed do not change on the interval $I_{r}^{N}$. The condition $\beta<\frac{1}{2}$ ensures that the two particles will escape the zone $\left\{\left|X_{u}^{N}-\bar{X}_{u}^{N}\right|<2 \varepsilon\right\}$ during the time interval $I_{r}^{N}$. Precisely, they are inside this zone at time $r$, and will after that have an increase (or decrease) of relative position larger than $C\left|V_{r}^{N}-\bar{V}_{r}^{N}\right|^{2} \geq C \varepsilon^{2 \beta}>4 \varepsilon$ if $\varepsilon$ is small enough.

Next, the previous bound by above on the relative velocities, implies that on the time interval $I_{r}^{N}$ the two particles can not stay $2 \varepsilon$-close too long. Such an encounter has a maximal duration of 
$\frac{8 \varepsilon}{\left|V_{r}^{N}-V_{r}^{N}\right|}$. Of course, by periodicity the same particles may have several close encounters on $I_{r}^{N}$ if their relative speed is large, but they can not have more than $C\left|V_{r}^{N}-\bar{V}_{r}^{N}\right|^{2}$. Summing up, we find than the total duration of this close encounters on the time interval $I_{r}^{N}$ is smaller than $C \varepsilon\left|V_{r}^{N}-\bar{V}_{r}^{N}\right|=C^{\prime} \varepsilon\left|I_{r}^{N}\right|$. Covering $A\left(Z_{.}^{N}, \bar{Z}^{N}\right)$ with such tile intervals, we end up with

$$
\left|A\left(Z^{N}, \bar{Z}^{N}\right)\right| \leq C \varepsilon|s-t| .
$$

Taking now the expectation, we get

$$
\left.\mathbb{E}_{\left(Z^{N}, \bar{Z}^{N}\right)}\left[\int_{s}^{t} W^{\prime}\left(X_{u}^{N}-\bar{X}_{u}^{N}\right) \chi_{\varepsilon}\left(X_{u}^{N}-\bar{X}_{u}^{N}\right)\left(1-\xi_{\varepsilon}\left(V_{u}^{N}-\bar{V}_{u}^{N}\right)\right) \partial_{v} \varphi\left(u, Z_{u}^{N}\right)\right] d u\right] \leq C \varepsilon|t-s|,
$$

uniformly in $N$. Remark that since the previous argument only requires that the velocities are almost surely Lipschitz (in time). So it also apply with $Z$., instead of $Z^{N}$ (See the last remark at the end of Step 2).

Step 5. The effect of close collision with small relative speed.

For the remaining term, we shall use a symmetry argument similar to the one used by Delort in its proof of existence of weak solutions to the 2D Euler equation Del91. In fact using the oddness of the interaction force $W^{\prime}$

$$
\begin{aligned}
& \left.\mathbb{E}_{\left(Z^{N}, \bar{Z}^{N}\right)}\left[\int_{s}^{t} W^{\prime}\left(X_{u}^{N}-\bar{X}_{u}^{N}\right) \chi_{\varepsilon}\left(X_{u}^{N}-\bar{X}_{u}^{N}\right) \xi_{\varepsilon}\left(V_{u}^{N}-\bar{V}_{u}^{N}\right) \partial_{v} \varphi\left(u, Z_{u}^{N}\right)\right] d u\right] \\
& =\mathbb{E}_{\left(Z^{N}, \bar{Z}^{N}\right)}\left[\int_{s}^{t} W^{\prime}\left(X_{u}^{N}-\bar{X}_{u}^{N}\right) \chi_{\varepsilon}\left(X_{u}^{N}-\bar{X}_{u}^{N}\right) \xi_{\varepsilon}\left(V_{u}^{N}-\bar{V}_{u}^{N}\right)\left(\partial_{v} \varphi\left(u, Z_{u}^{N}\right)-\partial_{v} \varphi\left(u, \bar{Z}_{u}^{N}\right)\right] d u\right] .
\end{aligned}
$$

But thanks to the two cut-off functions $\chi$ and $\xi$, we now have for all $(x, v, \bar{x}, \bar{v})$

$$
\chi_{\varepsilon}(x-\bar{x}) \xi_{\varepsilon}(v-\bar{v})\left|\partial_{v} \varphi(u, x, v)-\partial_{v} \varphi(u, \bar{x}, \bar{v})\right| \leq C\left\|\nabla^{2} \varphi\right\|_{\infty} \varepsilon^{\beta},
$$

and this implies that our last expectation is bounded by $C \varepsilon^{\beta}$, with a constant $C$ independent of $N$. That bound also apply when we replace $Z^{N}$ by $Z$.

2.3. Proof of Theorem 1.8. The proof of our central result is rather short.

Choose an optimal coupling of the random variables $Z_{0}^{1}$ and $Z_{0}^{2}$. We then couple the processes $Z^{1}$. and $Z^{2}$, using the previous coupling on the initial position independently of what happens after time 0 . This is relatively simple if there exists a unique trajectory starting from any initial condition. In the general case, the coupling is defined has the law on the couples $\left(Z_{.}^{1}, Z^{2}\right.$.) of trajectories such that for any smooth function $\varphi$ on $(\mathbb{T} \times \mathbb{R})^{2}$, and $\psi^{1}$ and $\psi^{2}$ on $C[0, \infty, \mathbb{T} \times \mathbb{R})$

$$
\mathbb{E}_{\left(Z^{1}, Z^{2}\right)}\left[\varphi\left(Z_{0}^{1}, Z_{0}^{2}\right) \psi^{1}\left(Z_{.}^{1}\right) \psi^{2}\left(Z_{.}^{2}\right)\right]=\mathbb{E}_{\left(Z_{0}^{1}, Z_{0}^{2}\right)}\left[\varphi\left(Z_{0}^{1}, Z_{0}^{2}\right)\right] \times \mathbb{E}_{Z^{1} \cdot}\left[\psi^{1}\left(Z_{.}^{1}\right) \mid Z_{0}^{1}\right] \times \mathbb{E}_{Z^{2}}\left[\psi^{2}\left(Z_{.}^{2}\right) \mid Z_{0}^{2}\right] .
$$

We also denote by $\left(\bar{Z}_{.}^{1}, \bar{Z}^{2}\right)$ an independent copy of the couple $\left(Z_{.}^{1}, Z_{.}^{2}\right)$. We can now differentiate $\mathbb{E}\left[\left|Z_{t}^{1}-Z_{t}^{2}\right|\right]$ with respect to time and get

$$
\begin{aligned}
\frac{d}{d t} \mathbb{E}\left[\left|Z_{t}^{1}-Z_{t}^{2}\right|\right] & \leq \mathbb{E}\left[\left|V_{t}^{1}-V_{t}^{2}\right|\right]+\mathbb{E}\left[\left|W^{\prime}\left(X_{t}^{1}-\bar{X}_{t}^{1}\right)-W^{\prime}\left(X_{t}^{2}-\bar{X}_{t}^{2}\right)\right|\right] \\
& \leq \sqrt{2} \mathbb{E}\left[\left|Z_{t}^{1}-Z_{t}^{2}\right|\right]+\mathbb{E}\left[\left|\operatorname{sign}\left(X_{t}^{1}-\bar{X}_{t}^{1}\right)-\operatorname{sign}\left(X_{t}^{2}-\bar{X}_{t}^{2}\right)\right|\right]
\end{aligned}
$$

To obtain the second line, we use the decomposition (1.2) of $W^{\prime}$, and the fact that $a+b \leq \sqrt{2\left(a^{2}+b^{2}\right)}$ for any $a, b \geq 0$.

To understand the remaining term, we remark first that $X_{t}^{1}-X_{t}^{2}$ and $\bar{X}_{t}^{1}-\bar{X}_{t}^{2}$ are "small" since the trajectories of respectively $\left(Z_{.}^{1}, Z_{.}^{2}\right)$ and $\left(\bar{Z}_{.}^{1}, \bar{Z}_{.}^{2}\right)$ are coupled in a more or less optimized way, while $X_{t}^{1}-\bar{X}_{t}^{1}$ is usually "large" since the trajectories of $Z^{1}$. and $\bar{Z}^{1}$. are independent (the same is true for $Z_{\text {. }}^{2}$ and $\bar{Z}_{\text {. }}^{2}$ ).

Neglecting the problem raised by the periodization of the sign function, which is not relevant, we see that they $X_{t}^{1}-\bar{X}_{t}^{1}$ and $X_{t}^{2}-\bar{X}_{t}^{2}$ may have different signs only if

$$
\left|X_{t}^{1}-\bar{X}_{t}^{1}\right| \leq\left|X_{t}^{1}-X_{t}^{2}\right|+\left|\bar{X}_{t}^{1}-\bar{X}_{t}^{2}\right| \leq 2 \max \left(\left|X_{t}^{1}-X_{t}^{2}\right|,\left|\bar{X}_{t}^{1}-\bar{X}_{t}^{2}\right|\right) .
$$


This condition is not symmetric with respect to $Z^{1}$ and $Z^{2}$, but this asymmetry is very important in order to obtain our weak-strong stability principle. Next

$$
\begin{aligned}
\mathbb{E}\left[\left|\operatorname{sign}\left(X_{t}^{1}-\bar{X}_{t}^{1}\right)-\operatorname{sign}\left(X_{t}^{2}-\bar{X}_{t}^{2}\right)\right|\right] & \leq \mathbb{E}\left[\mathbb{1}_{\left|X_{t}^{1}-\bar{X}_{t}^{1}\right| \leq 2 \max \left(\left|X_{t}^{1}-X_{t}^{2}\right|,\left|\bar{X}_{t}^{1}-\bar{X}_{t}^{2}\right|\right)}\right] \\
& \leq \mathbb{E}\left[\mathbb{1}_{\left|X_{t}^{1}-\bar{X}_{t}^{1}\right| \leq 2\left|X_{t}^{1}-X_{t}^{2}\right|}\right]+\mathbb{E}\left[\mathbb{1}_{\left.\left|X_{t}^{1}-\bar{X}_{t}^{1}\right| \leq 2\left|\bar{X}_{t}^{1}-\bar{X}_{t}^{2}\right|\right]}\right. \\
& \leq 2 \mathbb{E}\left[\mathbb{1}_{\left|X_{t}^{1}-\bar{X}_{t}^{1}\right| \leq 2\left|X_{t}^{1}-X_{t}^{2}\right|}\right] \\
& \leq 2 \mathbb{E}_{Z_{\cdot}^{1}, Z^{2}}\left[\mathbb{E}_{\bar{Z}^{1}}\left[\mathbb{1}_{\left|X_{t}^{1}-\bar{X}_{t}^{1}\right| \leq 2\left|X_{t}^{1}-X_{t}^{2}\right|}\right]\right] \\
& \leq 8\left\|\rho_{t}\right\|_{\infty} \mathbb{E}\left[\left|X_{t}^{1}-X_{t}^{2}\right|\right] .
\end{aligned}
$$

In the third line, we have used the symmetry in $Z ., \bar{Z}$.. The last line is obtained by using the independence of $\bar{Z}^{1}$ with respect to $\left(Z_{.}^{1}, Z_{.}^{2}\right)$ and the assumption that $\bar{X}_{t}^{1}$ has a bounded density $\rho_{t}$. All in all, we get that

$$
\frac{d}{d t} \mathbb{E}\left[\left|Z_{t}^{1}-Z_{t}^{2}\right|\right] \leq\left(\sqrt{2}+8\left\|\rho_{t}\right\|_{\infty}\right) \mathbb{E}\left[\left|Z_{t}^{1}-Z_{t}^{2}\right|\right]
$$

This concludes the proof.

2.4. Proof of Proposition 1.10. The proof is made in four steps. In the first one, we construct an approximating sequence. In the second one, we establish an a priori estimate on the density. In the third, we show that the approximating sequence has the Cauchy property. Finally, in the last one we prove that the limit process is a solution of the nonlinear ODE (1.8).

Step 1. Construction of an approximating sequence. We will as usual mollify the interaction force $W^{\prime}$. For $\varepsilon \in\left(0, \frac{1}{2}\right)$ we define a piecewise linear and continuous approximation of $W^{\prime}$ by

$$
W_{\varepsilon}(x):=\left\{\begin{array}{ll}
\frac{x^{2}-|x|}{2} & \text { if }|x| \in\left(\varepsilon, \frac{1}{2}\right) \\
-\left(\frac{1}{2 \varepsilon}-1\right) \frac{x^{2}}{2}-\frac{\varepsilon}{4} & \text { if } x \in[-\varepsilon, \varepsilon]
\end{array}, \quad-W_{\varepsilon}^{\prime}(x):=\left\{\begin{array}{ll}
-\frac{1}{2}-x & \text { if } x \in\left[-\frac{1}{2},-\varepsilon\right) \\
\frac{1}{2}-x & \text { if } x \in\left(\varepsilon, \frac{1}{2}\right) \\
\left(\frac{1}{2 \varepsilon}-1\right) x & \text { if } x \in[-\varepsilon, \varepsilon]
\end{array} .\right.\right.
$$

Remark that $W_{\varepsilon}^{\prime}$ is bounded by $\frac{1}{2}$ and has Lipschitz regularity with constant $\frac{1}{2 \varepsilon}$, and that $W_{\varepsilon}$ (as $W$ ) take values in $\left[-\frac{1}{8}, 0\right]$. We now consider the nonlinear ODE with the interaction potential $W_{\varepsilon}$. In others words, we look at solutions, in the sense of Definition (1.4), to

$$
\forall t \in[0,+\infty), \quad X_{t}^{\varepsilon}=X_{0}^{\varepsilon}+\int_{0}^{t} V_{s}^{\varepsilon} d s, \quad V_{t}^{\varepsilon}=V_{0}^{\varepsilon}-\int_{0}^{t} \mathbb{E}_{\bar{Z}^{\varepsilon}}\left[W_{\varepsilon}^{\prime}\left(X_{s}^{\varepsilon}-\bar{X}_{s}^{\varepsilon}\right)\right] d s,
$$

with initial data with law $f_{0}$. But the well-posedness of the associated "smoothed" Vlasov-Poisson equation is now well understood. The existence and uniqueness of solutions is known even if $f_{0}$ is a measure. In that setting, the first important works were done by Braun and Hepp BH77, and Dobrushin Dob79, and Neunzert and Wick NW80. Once we have a solution of the mollified VP equation (1.7), we can use the second part of Lemma 1.5 to obtain a process $Z^{\varepsilon}$. solution of the nonlinear ODE (2.17). But, a (maybe) better strategy will be to adapt on of the proofs mentioned above to the setting of nonlinear ODE. In fact, this will correspond to adapt our proof of the weak-strong stability result to the more simple case of Lipschitz interaction forces.

Step 2. An a priori estimates on $\left\|\rho_{t}^{\varepsilon}\right\|_{\infty}$. From the equations (2.17) satisfied by $Z^{\varepsilon}$, we get for all $t \in \mathbb{R}^{+}$

$$
\left|V_{t}^{\varepsilon}\right| \leq\left|V_{0}^{\varepsilon}\right|+\int_{0}^{t} \mathbb{E}_{\bar{Z}^{\varepsilon}[}\left[\left|W^{\prime}\left(X_{s}^{\varepsilon}-\bar{X}_{s}^{\varepsilon} \mid\right] d s \leq\right| V_{0}^{\varepsilon} \mid+\frac{t}{2} .\right.
$$

Since $f^{\varepsilon}$ is constant along the trajectories in our smooth setting, we get using (1.9) that

$$
\left|f_{t}^{\varepsilon}\left(X_{t}^{\varepsilon}, V_{t}^{\varepsilon}\right)\right|=\left|f_{0}\left(X_{0}^{\varepsilon}, V_{0}^{\varepsilon}\right)\right| \leq g_{0}\left(\left|V_{0}^{\varepsilon}\right|\right) \leq g_{0}\left(\left|V_{t}^{\varepsilon}\right|-\frac{t}{2}\right) \text {. }
$$


We used that $g_{0}$ is decreasing. Remark that the previous bound has no sense if $\left|V_{0}^{\varepsilon}\right| \leq \frac{t}{2}$. But in that case, we always have $\left|f_{t}^{\varepsilon}\left(X_{t}^{\varepsilon}, V_{t}^{\varepsilon}\right)\right| \leq\left\|f_{0}\right\|_{\infty}=g_{0}(0)$. So it will be consistent if we extend $g$ on $\mathbb{R}^{-}$, setting $g(x)=g(0)$ for all $x<0$. So for a trajectory such that $\left(X_{t}^{\varepsilon}, V_{t}^{\varepsilon}\right)=(x, v)$, we get with that convention

Integration on the variable $v$ leads to the bound

$$
f_{t}^{\varepsilon}(x, v) \leq g_{0}\left(|v|-\frac{t}{2}\right) .
$$

$$
\rho_{t}^{\varepsilon}(x)=\int_{\mathbb{R}} f_{t}^{\varepsilon}(x, v) d v \leq \int_{\mathbb{R}} g_{0}\left(|v|-\frac{t}{2}\right) d v \leq g(0) t+2 \int_{0}^{+\infty} g_{0}(v) d v .
$$

Step 3. The Cauchy property of the sequence $Z_{t}^{\varepsilon}$.

Thanks to the a priori bound (2.18), we may use a variant of the stability argument of Theorem 1.8 In fact, if we want to compare two processes $Z^{\varepsilon}$. and $Z^{\varepsilon^{\prime}}$ for two different $\varepsilon, \varepsilon^{\prime}$, we can basically apply the same strategy than in the proof of that theorem with $Z^{1}=Z^{\varepsilon}$ and $Z^{2}=Z^{\varepsilon^{\prime}}$. The only difference is that since $Z^{\varepsilon}$ is driven by the interaction potential $W_{\varepsilon}$ and $Z^{\varepsilon^{\prime}}$ is driven by $W_{\varepsilon^{\prime}}$, we will get an extra term (and also a less important additional $\varepsilon$ )

$$
\frac{d}{d t} \mathbb{E}\left[\left|Z_{t}^{\varepsilon}-Z_{t}^{\varepsilon^{\prime}}\right|\right] \leq\left(\sqrt{2}+8\left\|\rho_{t}^{\varepsilon}\right\|_{\infty}\right)\left(\mathbb{E}\left[\left|Z_{t}^{\varepsilon}-Z_{t}^{\varepsilon^{\prime}}\right|\right]+2 \varepsilon^{\prime}\right)+\mathbb{E}\left[\left|W_{\varepsilon}^{\prime}\left(X_{t}^{\varepsilon}-\bar{X}_{t}^{\varepsilon}\right)-W_{\varepsilon^{\prime}}^{\prime}\left(X_{t}^{\varepsilon}-\bar{X}_{t}^{\varepsilon}\right)\right|\right]
$$

But, from the definition of $W_{\varepsilon}^{\prime}$ and, we have

$$
\left|W_{\varepsilon}^{\prime}\left(X_{t}^{\varepsilon}-\bar{X}_{t}^{\varepsilon}\right)-W_{\varepsilon^{\prime}}^{\prime}\left(X_{t}^{\varepsilon}-\bar{X}_{t}^{\varepsilon}\right)\right| \leq \frac{1}{2} \mathbb{1}_{\left|X_{t}^{\varepsilon}-\bar{X}_{t}^{\varepsilon}\right| \leq \max \left(\varepsilon, \varepsilon^{\prime}\right)} .
$$

But, if we take the expectation and use the bound on $\rho_{t}^{\varepsilon}$ has in the proof of Theorem 1.8, we can bound the second term in the right hand side of (2.19) by

$$
\mathbb{E}\left[\left|W_{\varepsilon}^{\prime}\left(X_{t}^{\varepsilon}-\bar{X}_{t}^{\varepsilon}\right)-W_{\varepsilon^{\prime}}^{\prime}\left(X_{t}^{\varepsilon}-\bar{X}_{t}^{\varepsilon}\right)\right|\right] \leq\left\|\rho_{t}^{\varepsilon}\right\|_{\infty} \max \left(\varepsilon, \varepsilon^{\prime}\right),
$$

and finally get

$$
\frac{d}{d t} \mathbb{E}\left[\left|Z_{t}^{\varepsilon}-Z_{t}^{\varepsilon^{\prime}}\right|\right] \leq\left(\sqrt{2}+9\left\|\rho_{t}^{\varepsilon}\right\|_{\infty}\right)\left(\mathbb{E}\left[\left|Z_{t}^{\varepsilon}-Z_{t}^{\varepsilon^{\prime}}\right|\right]+2 \max \left(\varepsilon, \varepsilon^{\prime}\right)\right) .
$$

After slight modifications, this calculation will also lead to the stronger statement that there exists a constant $C_{t}$ such that

$$
\mathbb{E}\left[\sup _{s \leq t}\left|Z_{s}^{\varepsilon}-Z_{s}^{\varepsilon^{\prime}}\right|\right] \leq C_{t} \max \left(\varepsilon, \varepsilon^{\prime}\right)
$$

This implies that the $Z^{\varepsilon}$ form a Cauchy sequence for the weak topology on $\mathcal{P}(C([0,+\infty), \mathbb{T} \times \mathbb{R}))$. So in particular they converges towards a stochastic process $Z$..

Step 4. Characterization of the limit process $Z$.. In order to prove that the limit process $Z$. is a solution fo the nonlinear ODE (1.8) with the original interaction force $W^{\prime}$, we must for instance prove that for every time $t>0$,

$$
\mathbb{E}_{Z}\left[\left[\left|X_{t}-X_{0}-\int_{0}^{t} V_{s} d s\right|\right]=0, \quad \mathbb{E}_{Z} \cdot\left[\left|V_{t}-V_{0}+\int_{0}^{t} \mathbb{E}_{\bar{Z} \cdot}\left[W^{\prime}\left(X_{s}-\bar{X}_{s}\right)\right] d s\right|\right]=0 .\right.
$$

The first point is simple. For a fixed time $t$, the application that maps a trajectory $\tilde{Z}$. $\mapsto \mid \tilde{X}_{t}-\tilde{X}_{0}-$ $\int_{0}^{t} \tilde{V}_{s} d s \mid$ is continuous on $C([0,+\infty), \mathbb{T} \times \mathbb{R})$. It implies, see [Bil99, p.30] that the application that sent a process $Z$. to the first expectation in (2.20) is continuous. Since the expectation is already equals to zero for the approximated process $Z_{\text {. }}^{\varepsilon}$, we can pass to the limit and get the required equality.

For the second expectation, we have to be more careful because of the discontinuities in the interaction force. But if we define $F(x):=-\mathbb{E}_{Z}$. $\left[W^{\prime}\left(x-\bar{X}_{s}\right)\right]$ (where $Z$. stand for the fixed limit process), then the bound on the time marginals $\rho_{t}$ of $X$. implies that $F$ is continuous. Then, the application

$$
\tilde{Z} . \mapsto \mathbb{E}_{\tilde{Z}} \cdot\left[\left|V_{t}-V_{0}-\int_{0}^{t} F\left(X_{s}\right) d s\right|\right],
$$


is continuous. The problem is now that the previous expectation is not 0 for the approximated process. It will vanish for the approximated process only if we replace $F$ by $F^{\varepsilon}(x)=-\mathbb{E}_{\bar{Z}^{\varepsilon}}\left[W_{\varepsilon}^{\prime}\left(x-\bar{X}_{s}\right)\right]$. But even if it is not emphasize, we have proved in the second step that $F^{\varepsilon}$ is a Cauchy sequence in $L^{1}(\mathbb{T})$, and in particular it converges strongly in the $L^{1}$-norm towards $F$. This and the uniform bound on the density allows to pass to the limit and we obtain that the second expectation in (2.20) also vanishes. This concludes the proof.

\section{REFERENCES}

[Amb04] Luigi Ambrosio. Transport equation and Cauchy problem for BV vector fields. Invent. Math., 158(2):227$260,2004$.

[Amb08] Luigi Ambrosio. Transport equation and Cauchy problem for non-smooth vector fields. In Calculus of variations and nonlinear partial differential equations, volume 1927 of Lecture Notes in Math., pages 1-41. Springer, Berlin, 2008.

[BH77] W. Braun and K. Hepp. The Vlasov dynamics and its fluctuations in the $1 / N$ limit of interacting classical particles. Comm. Math. Phys., 56(2):101-113, 1977.

[Bil99] Patrick Billingsley. Convergence of probability measures. Wiley Series in Probability and Statistics: Probability and Statistics. John Wiley \& Sons Inc., New York, second edition, 1999. A Wiley-Interscience Publication.

[Boi11] E. Boissard. Problèmes d'interaction discret-continu et distances de Wasserstein. PhD thesis, Université de Toulouse III, 2011.

[Bos05] M. Bostan. Existence and uniqueness of the mild solution for the 1D Vlasov-Poisson initial-boundary value problem. SIAM J. Math. Anal., 37(1):156-188, 2005.

[Bou01] François Bouchut. Renormalized solutions to the Vlasov equation with coefficients of bounded variation. Arch. Ration. Mech. Anal., 157(1):75-90, 2001.

$\left[\mathrm{CDF}^{+} 11\right]$ J. A. Carrillo, M. DiFrancesco, A. Figalli, T. Laurent, and D. Slepčev. Global-in-time weak measure solutions and finite-time aggregation for nonlocal interaction equations. Duke Math. J., 156(2):229-271, 2011.

[CK80] Jeffery Cooper and Alexander Klimas. Boundary value problems for the Vlasov-Maxwell equation in one dimension. J. Math. Anal. Appl., 75(2):306-329, 1980.

[Del91] Jean-Marc Delort. Existence de nappes de tourbillon en dimension deux. J. Amer. Math. Soc., 4(3):553-586, 1991.

[DFVar] F. Delarue, F. Flandoli, and D. Vincenzi. Noise prevents collapse of vlasov-poisson point charges. Commun. Pure Appl. Math., To appear.

[DL89] Ronald J. DiPerna and Pierre-Louis Lions. Ordinary differential equations. Invent. Math, 98:511-547, 1989.

[Dob79] R. L. Dobrušin. Vlasov equations. Funktsional. Anal. i Prilozhen., 13(2):48-58, 96, 1979.

[Fig08] Alessio Figalli. Existence and uniqueness of martingale solutions for SDEs with rough or degenerate coefficients. J. Funct. Anal., 254(1):109-153, 2008.

[Fil88] A. F. Filippov. Differential equations with discontinuous righthand sides, volume 18 of Mathematics and its Applications (Soviet Series). Kluwer Academic Publishers Group, Dordrecht, 1988. Translated from the Russian.

[Guo95] Yan Guo. Singular solutions of the Vlasov-Maxwell system on a half line. Arch. Rational Mech. Anal., 131(3):241-304, 1995.

[Hén82] M. Hénon. Vlasov equation? Astronom. and Astrophys., 114(1):211-212, 1982.

[HM12] Maxime Hauray and Stéphane Mischler. On Kac's chaos and related problems. To appear in JFA. http://www.arxiv.org/pdf/1205.4518, 2012.

[LMR10] Simon Labrunie, Sandrine Marchal, and Jean-Rodolphe Roche. Local existence and uniqueness of the mild solution to the 1D Vlasov-Poisson system with an initial condition of bounded variation. Math. Methods Appl. Sci., 33(17):2132-2142, 2010.

[McK66] H. P. McKean, Jr. A class of Markov processes associated with nonlinear parabolic equations. Proc. Nat. Acad. Sci. U.S.A., 56:1907-1911, 1966.

[McK67] H. P. McKean, Jr. Propagation of chaos for a class of non-linear parabolic equations. In Stochastic Differential Equations (Lecture Series in Differential Equations, Session 7, Catholic Univ., 1967), pages 41-57. Air Force Office Sci. Res., Arlington, Va., 1967.

[Mél96] Sylvie Méléard. Asymptotic behaviour of some interacting particle systems; McKean-Vlasov and Boltzmann models. In Probabilistic models for nonlinear partial differential equations (Montecatini Terme, 1995), volume 1627 of Lecture Notes in Math., pages 42-95. Springer, Berlin, 1996.

[MM11] S. Mischler and C. Mouhot. Kac's Program in Kinetic Theory. ArXiv e-prints, July 2011.

[MMZ94a] Andrew J. Majda, George Majda, and Yu Xi Zheng. Concentrations in the one-dimensional Vlasov-Poisson equations. I. Temporal development and non-unique weak solutions in the single component case. Phys. D, 74(3-4):268-300, 1994. 
[MMZ94b] Andrew J. Majda, George Majda, and Yu Xi Zheng. Concentrations in the one-dimensional Vlasov-Poisson equations. II. Screening and the necessity for measure-valued solutions in the two component case. Phys. D, 79(1):41-76, 1994.

[NW80] H. Neunzert and J. Wick. The convergence of simulation methods in plasma physics. In Mathematical methods of plasmaphysics (Oberwolfach, 1979), volume 20 of Methoden Verfahren Math. Phys., pages 271286. Lang, Frankfurt, 1980.

[Ste70] Elias M. Stein. Singular integrals and differentiability properties of functions. Princeton Mathematical Series, No. 30. Princeton University Press, Princeton, N.J., 1970.

[Szn91] Alain-Sol Sznitman. Topics in propagation of chaos. In École d'Été de Probabilités de Saint-Flour XIX1989, volume 1464 of Lecture Notes in Math., pages 165-251. Springer, Berlin, 1991.

[Tro86] M. Trocheris. On the derivation of the one-dimensional Vlasov equation. Transport Theory Statist. Phys., 15(5):597-628, 1986.

[Vil03] Cédric Villani. Topics in optimal transportation, volume 58 of Graduate Studies in Mathematics. American Mathematical Society, Providence, RI, 2003.

[ZM94] Yu Xi Zheng and Andrew Majda. Existence of global weak solutions to one-component Vlasov-Poisson and Fokker-Planck-Poisson systems in one space dimension with measures as initial data. Comm. Pure Appl. Math., 47(10):1365-1401, 1994.

M. Hauray: LATP, Université D'Aix-Marseille \& CNRS UMR 7353, 13453 Marseille Cedex 13, France.

E-mail address: maxime.hauray@univ-amu.fr 